\title{
Enteroparasitoses e aspectos socioeconômicos em pacientes alcoolistas
}

\author{
Intestinal parasites and socioeconomic aspects of alcoholic patients
}

\author{
Cíntia de Lima Oliveira ${ }^{1}$, Marina Morena Brito Farias ${ }^{1}$, Nilo Manoel Pereira Vieira Barreto ${ }^{2}$, Joelma \\ Nascimento de Souza ${ }^{3}$, Larissa Mota Sampaio ${ }^{4}$, Márcia Cristina Aquino Teixeira ${ }^{5}$, Neci Matos Soares ${ }^{6 *}$ \\ ${ }^{1}$ Mestranda do Programa de Pós Graduação em Processos Interativos dos Órgãos e Sistemas, UFBA. \\ ${ }^{2}$ Doutorando do Programa de Pós Graduação em Processos Interativos dos Órgãos e Sistemas, UFBA. ${ }^{3}$ Pós \\ doutoranda Escola Bahiana de Medicina e Saúde Pública ${ }^{4}$ Estudante curso de farmácia, UFBA, ${ }^{5}$ Professora Adjunta, \\ Faculdade de Farmácia, UFBA; ${ }^{6}$ Professora Titular, Faculdade de Farmácia, UFBA.
}

\begin{abstract}
Resumo
Introdução: as enteroparasitoses constituem um problema de saúde pública que atinge as populações mais vulneráveis. $\mathrm{O}$ alcoolismo é um problema que afeta milhões de pessoas em todo o mundo, apresenta efeitos nocivos à saúde dos indivíduos e predispõe à infecção por S. stercoralis e às formas mais graves da estrongiloidíase. Objetivo: avaliar a prevalência da infecção das enteroparasitoses, bem como fatores socioeconômicos e sanitários de pacientes alcoolistas atendidos em um centro de tratamento especializado em Salvador, Bahia. Metodologia: trata-se de um estudo transversal, realizado com pacientes alcoolistas ( $n=277$ ), do sexo masculino, atendidos em um centro de tratamento entre julho de 2014 e junho de 2017. O estudo foi realizado através da aplicação de um questionário estruturado para obtenção de dados socioeconômicos, demográficos e sanitários, sendo solicitadas, ainda, pelo menos duas amostras de fezes em dias alternados. O diagnóstico parasitológico foi realizado pelos métodos de sedimentação espontânea, Baermann-Moraes e cultura em placa de ágar. Resultados: a média de idade dos pacientes inclusos foi de $44,8 \pm 9,5$ anos. Verificouse que $35,7 \%$ dos pacientes estavam infectados com algum enteroparasito e, desses, 30,3\% eram monoparasitados. Os parasitos mais frequentes foram S. stercoralis, Endolimax nana e ancilostomídeos, correspondendo 16,6\%, 11,2\% e 5,8\%, respectivamente. Conclusão: dessa forma, S. stercoralis foi o parasito mais frequente, e a associação entre a infecção por esse parasito e o alcoolismo pode levar à estrongiloidíase grave. A elevada frequência de enteroparasitoses nesses pacientes, principalmente por protozoários, deve-se à ausência de higiene pessoal, pois esses indivíduos são suscetíveis à infecção oral fecal. A implementação de medidas de saneamento básico e de campanhas de conscientização para prevenção de infecções parasitárias é imprescindível para a saúde das populações mais vulneráveis.
\end{abstract}

Palavras-chave: Aspectos socioeconômicos. Alcoolistas. S. stercoralis

\begin{abstract}
Introduction: intestinal parasitoses are a public health issue that notably affects vulnerable populations. Alcoholism is a problem that affects millions worldwide by causing damaging effects to the health of individuals as it predisposes them to that infection through stercralisàs and more evolved forms of strongyloidiasis. Objective: evaluate the prevalence of intestinal parasite infections as well as socioeconomic and sanitation factors in a population of alcoholic patients at a specialized treatment facility in Salvador, Bahia. Methodology: this cross-sectional study was carried out with male alcoholic patients $(n=277)$ at a treatment facility from July/2014 to June/2017. The research data were collected through a structured questionnaire aimed to obtain socioeconomic, demographic and sanitation information. Stool tests were ordered in alternate days. The parasitological diagnosis was based on methods of spontaneous sedimentation, Baermann-Moraes and Agar plate growth medium. Results: the patients' average age was 44,8 $\pm 9,5$ years. A total of $35 \%$ of the patients was infected with a type of enteroparasite, of whom $30,3 \%$ was mono-infected. The most commonly found parasites were S. stercoralis, Endolimax nana and hookworm, corresponding to 16,6\%, 11,2\% e 5,8\%, respectively. Conclusion: as such, S. Stercoralis was the most frequent parasite in this population and the association between the infection by this parasite and alcoholism may lead to severe strongyloidiasis. The high rates of enteroparasitism in those patients, particularly protozoa, are due to the lack of adequate personal hygiene, given those individuals are prone to acquire infections transmitted through the oral fecal route. An implementation of public health sanitation measures and the launch of public awareness campaigns in order to prevent parasite infections are vital to the health of vulnerable populations.
\end{abstract}

Kewords: Social economic factors. Alcoholic patients. S. stercoralis

\section{INTRODUÇÃO}

As parasitoses intestinais continuam sendo um grave problema de saúde pública, principalmente nos países subdesenvolvidos onde a falta de investimentos

Correspondente/Corresponding: *Neci Matos Soares - Instituto de Ciências da Saúde - UFBA - End: Av. Reitor Miguel Calmon S/N sala 413 Canela, Salvador-Ba CEP: 40231-300 - Tel: (71) 99975-3308 - E-mail: necisoares@gmail.com em educação e saneamento básico leva a um alto grau de contaminação e disseminação das enteroparasitoses (MELO et al., 2004; NEVES et al., 2011). Outros fatores, como idade, hábitos de higiene e outras comorbidades podem contribuir significativamente para a aquisição destes parasitos (MACHADO et al., 1999; ROCHA et al., 2001).

Segundo a Organização Mundial da Saúde (2016), as parasitoses, constituem as doenças mais comuns do 
mundo, e podem ser encontradas tanto em zonas rurais quanto em áreas urbanas, com uma variação que depende da espécie parasitária, do ambiente e das condições do hospedeiro. Com relação às helmintíases, mais de 1,5 bilhões pessoas em torno de $24 \%$ da população mundial estão infectadas, com uma maior incidência entre as áreas tropicais e subtropicais. Em municípios com baixo índice de desenvolvimento humano (IDH), valores de prevalência de geohelmintos variam entre 2 e $36 \%$ (BRASIL, 2012).

Estima-se, que 200 e 500 milhões de indivíduos estão infectados com Giardia duodenalis e Entamoeba histolytica, respectivamente (BAPTISTA et al., 2006; FONSECA; PEÑUELA; BOTERO, 2009; LODO et al., 2010; WHO, 2005). No Brasil, por sua ampla diversidade geográfica, climática, econômica e social, encontra-se uma grande variedade de enteropatógenos, principalmente os que causam diarreias (SCHNACK et al., 2003).

São diversos os danos que os parasitas podem ocasionar à saúde dos portadores, tais como: alterações no equilíbrio nutricional, sangramento e obstrução intestinal, prolapso retal e diarreia, entre outros, sendo as manifestações clínicas normalmente proporcionais à carga parasitária, podendo levar o indivíduo ao óbito (COLLI et al., 2014; SANTOS; MERLINI, 2010). Além de prejuízos cognitivos e econômicos (COLLI et al., 2014; OMS, 2015).

As populações que vivem em precárias condições de saneamento e os pacientes com alterações metabólicas e imunológicas tendem a ser mais suscetíveis a doenças parasitárias, como os indivíduos imunocomprometidos, alcoolistas e transplantados (BUONFRATE et al., 2012; KEISER; NUTMAN, 2004). O alcoolismo é um problema que afeta milhões pessoas em todo o mundo. Segundo a Organização Mundial de Saúde (WHO, 2004), estima-se que dois bilhões de pessoas consomem álcool e 76,3 milhões apresentam os efeitos nocivos de seu uso, o que representa um sério problema de saúde pública. No Brasil, segundo o centro brasileiro de informações sobre drogas psicotrópicas, o consumo que causa dependência atinge $9,4 \%$ de indivíduos nas principais cidades do país (GALDURÓZ; CAETANO, 2004), sendo essa a principal causa de mais de $10 \%$ de toda a morbidade e mortalidade no país (MELONI; LARANJEIRA, 2004). Ademais, o consumo crônico do álcool resulta na modulação da resposta imune na defesa de organismos patogênicos, como $S$. stercoralis e na predisposição para formas mais graves da estrongiloidíase (DE-OLIVEIRA et al., 2002; SILVA, et al., 2015; TEIXEIRA et al., 2010).

Diante da diversidade dos parasitos e dos vários fatores que influenciam nas infecções parasitárias o objetivo deste estudo é avaliar a prevalência da infecção das enteroparasitoses e fatores socioeconômicos e sanitários de pacientes alcoolistas atendidos em um centro de tratamento especializado em Salvador, Bahia.

\section{METODOLOGIA}

Trata-se de um estudo transversal com pacientes al- coólicos, maiores de 18 anos do sexo masculino ( $n=277)$, atendidos no Centro de Acolhimento e Tratamento de Alcoolistas (CATA) das Obras Sociais Irmã Dulce, instituição localizada em Salvador (BA) no período entre julho de 2014 a Junho de 2017.

Os dados socioeconômicos, demográficos e sanitários foram obtidos através da aplicação de um questionário semi-estruturado. 0 diagnóstico parasitológico foi realizado pelos métodos de Sedimentação Espontânea, Baermann-Moraes e Cultura em Placa de Ágar com no mínimo duas amostras em dias alternados.

O estudo foi aprovado pelo Comitê de Ética em Pesquisa da Escola de Enfermagem da Universidade Federal da Bahia sob o número 367.464 e respeitou a Resolução № 466, de 12 de dezembro de 2012 do Conselho Nacional de Saúde. Os participantes assinaram o Termo de Consentimento Livre e Esclarecido. Os dados foram tabulados e analisados através do IBM SPSS software (versão 19.0 para Windows, Estados Unidos da América). Variáveis contínuas foram expressas como média (desvio padrão) e variáveis categóricas em percentual.

\section{RESULTADOS}

A média de idade dos 277 pacientes alcoolistas participantes do estudo foi de $44,8 \pm 9,8$, concentrados predominantemente na faixa etária de 41 a 50 , com uma variação de 26 a 75 anos. A maior parte dos alcoolistas - 81,2\% (225/277) - afirmou fazer ingestão diária de bebidas alcoólicas, e os demais, $18,8 \%$ (53/277), de uma a quatro vezes por semana (Tabela 1 ).

O total de indivíduos parasitados foi de $35,7 \%$ (99/277). Desses, 30,3\% (84/277) eram monoparasitados e 5,4\% (15/277) poliparasitados.

A frequência da infecção por $S$. Stercoralis em indivíduos alcoolistas foi de 16,6\% (46/277). As demais enteroparasitoses foram por Endolimax nana (11,2\%), ancilostomídeos (5,8\%), Entamoeba coli (3,6\%), Schistosoma mansoni (2,9\%) e Giardia duodenalis (1,8\%). (Tabela 1$)$.

Tabela 1 - Frequência de parasitos intestinais em pacientes alcoolistas, atendidos no Centro de Acolhimento e Tratamento de Alcoolistas (CATA) $(n=277)$

\begin{tabular}{lc}
\hline Parasito & No de amostras positivas (\%) \\
\hline Poliparasitados & $15(5,4)$ \\
Monoparasitados & $84(30,3)$ \\
Parasitados & $99(35,7)$ \\
Sem infeção & $178(64,3)$ \\
Helmintos & \\
Strongyloides stercoralis & $46(16,6)$ \\
Ancilostomídeos & $16(5,8)$ \\
Ascaris lumbricoides & $1(0,4)$ \\
Schistosoma mansoni & $8(2,9)$ \\
Trichuristrichiura & $2(0,7)$ \\
Protozoários &
\end{tabular}


Enteroparasitoses e aspectos socioeconômicos em pacientes alcoolistas

\begin{tabular}{lc}
\hline Parasito & № de amostras positivas (\%) \\
\hline lodamoebabutschilli & $1(0,4)$ \\
Giardia duodenalis & $5(1,8)$ \\
Entamoebahistolytica/ díspar & $2(0,7)$ \\
Entamoeba coli & $10(3,6)$ \\
Endolimax nana & $31(11,2)$ \\
\hline
\end{tabular}

Fonte: Autoria própria

Dos pacientes avaliados, 68,6\% (190/277) residiam na cidade de Salvador, enquanto $28,9 \%(10 / 277)$ residiam no interior do estado da Bahia e 2,5\% (0,90/277), em outras cidades do país. De acordo com os dados socioeconômicos 63,5\% (176/277) dos pacientes alcoolistas apresentavam renda mensal inferior ou igual a um salário mínimo variando de $1,4 \pm 0,79$. Com relação à escolaridade, cerca de $39,4 \%(109 / 277)$ estudaram da $5^{a}$ a $8^{\underline{a}}$ série, $23,1 \%$ (64/277) estudaram da 1a a 4 a série, tendo completado o ensino médio somente $18,0 \%$ (50/277) dos entrevistados. (Tabela 2). A maioria dos pacientes possui água tratada, 98,8\% (257/277), e 49,4\% (137/277) deles fazem ingestão de água filtrada. E ainda 94,9\% (263/277) possuem rede de esgoto, $91,3 \%$ (253/277) residem em ruas pavimentadas, $96,8 \%(268 / 277)$ possuem banheiro em casa e $87,7 \%$ (243/277) são beneficiados diariamente pelo serviço coleta de lixo. Quanto aos hábitos de vida, $18,41 \%$ (51/277) relataram o hábito de andar descalço e 57,4\% (159/277) são tabagistas (Tabela 2).

Tabela 2 - Características demográficas e socioeconômicas dos pacientes alcoolistas ATTTTT $(n=277)$

\begin{tabular}{lr}
\hline Variáveis & \multicolumn{1}{|c}{$\mathbf{N}(\%)$} \\
\hline- Idade (anos)* & $18(6,5 \%)$ \\
$20-30$ & $83(29,9 \%)$ \\
$31-40$ & $96(34,6 \%)$ \\
$41-50$ & $67(24,1 \%)$ \\
$51-60$ & $13(4,9 \%)$ \\
$>60$ & \\
- Município de residência & $190(68,6 \%)$ \\
Salvador & $80(28,9 \%)$ \\
Interior do Estado & $7(2,5 \%)$ \\
Outras cidades & \\
- Ingestão de álcool & $225(81,2 \%)$ \\
Todos os dias & $36(13,0 \%)$ \\
1-4 vezes por semana & $16(5,8 \%)$ \\
Não informou & \\
- Grau de escolaridade & $14(5,1 \%)$ \\
Nenhum & $64(23,1 \%)$ \\
1à à 4ạ série & $109(39,4 \%)$ \\
5a à 8a série & $37(13,4 \%)$ \\
Ensino médio incompleto & $50(18,1 \%)$ \\
Ensino médio completo & $1(0,4 \%)$ \\
Superior & $2(0,7 \%)$ \\
Não informou & $176(63,5 \%)$ \\
- Renda mensal &
\end{tabular}

Rev. Ciênc. Méd. Biol., Salvador, v. 17, n. 3, p. 345-349, set./dez. 2018

\begin{tabular}{lr}
\hline Variáveis & \multicolumn{1}{c}{ N (\%) } \\
\hline 2 salários mínimos & $76(27,4 \%)$ \\
3 salários mínimos & $16(5,8 \%)$ \\
4 salários mínimos & $6(2,2 \%)$ \\
> 4 salários mínimos & $3(1,1 \%)$ \\
- Aspectos sanitários & \\
Possui rede de esgoto (sim) & $263(94,9 \%)$ \\
Ruas são pavimentadas (sim) & $253(91,3 \%)$ \\
Coleta de lixo diariamente (sim) & $243(87,7 \%)$ \\
Possui água encanada (sim) & $257(98,8 \%)$ \\
Possui banheiro em casa (sim) & $268(96,8 \%)$ \\
Consumo de água filtrada (sim) & $137(49,4 \%)$ \\
Hábitos de vida & \\
Anda descalço (sim) & $51(18,41 \%)$ \\
Tabagista & $159(57,4 \%)$ \\
Consomem cachaça & $205(74,0 \%)$ \\
Consomem cerveja & $57(20,7 \%)$ \\
\hline
\end{tabular}

* Média de idade \pm SD (anos) 44,8 9,8 / Variação da idade $26 \sim 75$

Fonte: Autoria própria

\section{DISCUSSÃO}

Neste estudo, foi observado que $35,7 \%$ dos pacientes estavam infectados com um ou mais parasitos intestinais. O parasito mais frequente foi $S$. stercoralis $(16,6 \%)$, o que corrobora outros estudos em que essa frequência variou de 20 a 23,5\% (MARQUES et al., 2010; SILVA et al., 2015; ZAGO-GOMES et al., 2002). De modo geral, poucos estudos avaliaram a frequência dos enteroparasitos em alcoolistas. Zago-Gomes et al. (2002) demostraram que $35,3 \%$ deles apresentaram infecção apenas por nematódeos. Silva et al. (2015) evidenciaram a prevalência de poliparasitados em $13,3 \%$, sendo que $S$. stercoralis e ancilostomídeos apresentaram as maiores frequências, $23,5 \%$ e $11,7 \%$, respectivamente.

Pacientes alcoólicos infectados pelo $S$. stercoralis são mais susceptíveis à hiperinfecção. A ingestão prolongada de álcool exerce efeito sob o eixo hipotálamo-pituitária-adrenal, elevando os níveis dos metabólitos de corticosteroides endógenos, que por sua semelhança com a ecdisona, o hormônio que faz a regulação da fecundidade das fêmeas partenogenéticas, estimula a transformação das larvas favorecendo a autoinfecção e consequentemente, a hiperinfecção. Recentemente, num trabalho publicado pelo nosso grupo, foi demostrado que os níveis de cortisol endógeno estão correlacionados positivamente com a carga parasitária dos indivíduos alcoólicos infectados com $S$. stercoralis. Os altos níveis de corticosteroides endógenos também suprimem a resposta imunológica dos pacientes e induzem a modificação do perfil de resposta imune, o que pode contribuir para a sobrevivência do parasito e agravamento da estrongiloidíase. (De SOUZA et al., 2014; TEIXEIRA et al., 2016; THAYER et al., 2006).

De modo geral, as condições socioeconômicas e ambientais em que vive o indivíduo são determinantes para a obtenção de várias infecções por enteroparasitos, ressaltando-se as condições de moradia, abastecimento 
de água, destino do esgoto, lixo e renda salarial, como também a ausência de higiene pessoal. (CAMELLO et al., 2016; SHIFERAW; MENGISTU, 2015). Neste estudo, foi encontrada uma elevada frequência de infecções por enteroparasitoses em indivíduos alcoólicos, 30,3\% dos indivíduos estavam monoparasitados e 5,4\% poliparasitados. (Tabela 1).

Embora grande maioria dos pacientes tenha acesso a abastecimento de água encanada $(98,8 \%)$ e rede de esgoto (94,9\%), com índices superiores aos brasileiros segundo o IBGE (2015): água encanada, 85,4\%, rede de esgotos, 65,3\% (IBGE, 2015) -, geralmente os pacientes alcoólicos são suscetíveis à infecção oral fecal, que é a via por meio da qual ocorre a transmissão da maioria dos protozoários. (SILVA et al., 2015). Para a infecção por ancilostomídeos e S. stercoralis, cuja principal forma de infecção é a penetração das larvas filarióides através da pele, andar descalço, ter contato direto com solo e com conteúdo infectado são as principais vias de transmissão, fatores que também podem estar relacionados com os hábitos dos pacientes alcoolistas.

Diante do exposto, $S$. stercoralis foi o parasito mais frequente, e a associação entre a infecção por esse parasito e o alcoolismo pode levar à estrongiloidíase grave. Os pacientes em uso crônico de álcool, quando não tratados, perpetuam a infecção pelo mecanismo de autoinfecção e permanecem como uma fonte de contaminação. A elevada frequência de enteroparasitoses nesses pacientes, principalmente protozoários, deve-se à ausência de higiene pessoal, pois esses indivíduos são suscetíveis à infecção oral fecal. A implementação de medidas de saneamento básico e de campanhas de conscientização para prevenção de infecções parasitárias é imprescindível para a saúde das populações mais vulneráveis.

\section{REFERÊNCIAS}

BAPTISTA, S. C. et al. Análise da incidência de parasitoses intestinais no município de Paraíba do Sul, RJ. Rev. bras. anal. clín., Rio de Janeiro, v. 38, n. 4, p. 271-273, 2006.

BRASIL Ministério da Saúde. Plano integrado de ações estratégicas de eliminação da hanseníase, filariose, esquistossomose e oncocercose como problema de saúde pública, tracoma como causa de cegueira e controle das geohelmintíases. Brasília:MS,2012.

BUONFRATE, D. et al. Imported strongyloidiasis: epidemiology, presentations, and treatment. Curr. Infect. Dis. Rep., Philadelphia, v. 14, n. 3, p. 256-262, 2012 .

CAMELLO, J. T. et al. Prevalence of intestinal parasites among schoolchildren and household sanitation in the urban area of Caxias do Sul, State of Rio Grande do Sul, Brazil. Sci. med., Porto Alegre, v. 26, n. 1, p. 21716, 30 mar. 2016.

COLLI, C. M. et al. Prevalence and risk factors for intestinal parasites in food handlers, southern Brazil. Int. J. environ. health res., Abingdon, $v$. 24, n. 5, p. 450-458, 2014.

DE-OLIVEIRA, L. C. et al. Frequency of Strongyloide stercoralis infection in alcoholics. Mem. Inst. Oswaldo Cruz, Rio de Janeiro, v. 97, n. 1, p.119-121, 2002.
DE SOUZA, J. N. et al. Recurrence of strongyloides stercoralis infection in a patient with Hansen's disease: a case report. Lepr. rev., London, v. 85, n. 1, p. $58-62,2014$

FONSECA, J. C.; PEÑUELA, R. M.; BOTERO, A. M. Parasitosis intestinal em niños de zonas palúdicas de Antioquia (Colombia). latreia, Medellín, v.22, n.1, jan./mar. 2009.

GALDURÓZ, J. C. F., CAETANO, R. Epidemiologia do uso de álcool no Brasil [Epidemiology of alcohol use in Brazil]. Rev. bras. psiquiatr., São Paulo, v.26, Supl. 1, p. 3-6, 2004.

IBGE. Instituto Brasileiro de Geografia e Estatística. 2015. Disponível em: https://www.ibge.gov.br/ Acesso em: 02 ago. 2018.

LODO, M. et al. Prevalência de endoparasitas em município do interior paulista. Rev. bras. crescimento desenvolv. hum., São Paulo, v. 20, n.3, p. 769-777, mar. 2010.

KEISER, P.; NUTMAN, T. B. Strongyloides stercoralis in the immunocompromised population. Clin. microbiol. rev., Washington, v. 17, p. 208-217, 2004.

MACHADO, R. C. et al. Giardíase e Helmintíases em crianças de creches e escolas de 10 e $2 \circ$ graus (públicas e privadas) da cidade de Mirassol (SP, Brasil). Rev. Soc. Bras. Med. Trop., Brasília, v. 32, n.5, p. 697-704, 1999.

MARQUES, C. C. et al. Alcoholism and Strongyloides stercoralis: daily ethanol ingestion has a positive correlation with the frequency of strongyloides larvae in the stools. PLOS negl. trop. dis., San Francisco, v. 4, n. 6, p. e717, 2010. DOI: 10.1371/journal.pntd.0000717.

MELO, M. C. B. et al. Parasitoses intestinais. Rev. méd. Minas Gerais, Belo Horizonte, v. 14, n. 3, p.12, 2004.

MELONI, J. N.; LARANJEIRA, R. Custo social e de saúde do consumo do álcool. Rev. bras. psiquiatr., São Paulo, v. 26, Supl I, p. 7-10, 2004.

NEVES, D. P. et al. Parasitologia humana. 12. Ed. São Paulo: Atheneu, 2011.

ORGANIZAÇÃO MUNDIAL DA SAÚDE (OMS) 2015. Disponível em: <| http://www.who.int/about/es/>. Acesso em: 01 jun. 2018.

ROCHA, R. S. et al. Avaliação das esquistossomoses e de outras parasitoses intestinais, em escolares do município de Bambuí, Minas Gerais, Brasil. Rev. Soc. Bras Med. Trop., Brasília, v.39, p. 99-101, 2001.

SAGNUANKIAT, S. et al. Health status of immigrant children and environmental survey of child daycare centers in Samut Sakhon Province, Thailand. J. immigr. minor health, New York, , v. 18, n. 1, p. 21-27, 2016.

SANTOS S. A.; MERLINI L. S. Prevalence of enteroparasitosis in the population of Maria Helena, Parana State. Ciênc. Saúde Colet., Rio de Janeiro, v. 15, p. 899-905, 2010.

SCHNACK, F. J. et al. Enteropathogens associated with diarrheal disease in infants ( $<5$ years old) in a population sample in Greater Metropolitan Criciuma, Santa Catarina State, Brazil. Cad. Saúde Pública, Rio de Janeiro, v.19, p. 1205-1258, 2003.

SHIFERAW, M. B.; MENGISTU, A. D. Helminthiasis: hookworm infection remains a public health problem in Dera District, South Gondar, Ethiopia. PLos ONE, San Francisco, v. 10, n. 12, 2015.

SILVA, M. L. S. et al. Association between Strongyloides stercoralis infection and cortisolsecretion in alcoholic patients. Acta trop., Basel, v. 154, p. 133-138, Nov. 2015.

TEIXEIRA, M. C. et al. Asymptomatic Strongyloides stercoralis hyperinfection in an alcoholic patient with intense. J. parasitol., Lawrence, v. 96, n.4, p. 833-835, 2010. 
TEIXEIRA, M. C. A. et al. Strongyloides stercoralis infection in alcoholic patients. BioMed Res. Int., New York, v. 2016, p. e4872473, Dec. 2016.

THAYER, J. F. et al. Alcohol use, urinary cortisol, and heart rate variability in apparently healthy men: evidence for impaired inhibitory control of the HPA axis in heavy drinkers. Int j. psychophysiol., Amsterdam, v.59, n. 3, p. 244-250, Mar. 2006.
WORLD HEALTH ORGANIZATION (WHO). Soil-transmitted helminth infections. 2004.

WORLD HEALTH ORGANIZATION (WHO). Soil-transmitted helminth infections. 2005.

ZAGO-GOMES, M. P. et al. Prevalence of intestinal nematodes in alcoholic patients. Rev. Soc. Bras Med. Trop., Brasília, v. 35, p. 571-574, 2002.

Submetido em: 19/11/2018

Aceito em: 29/11/2018 